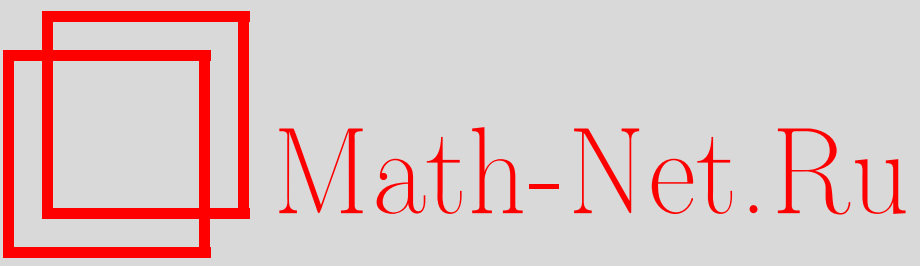

Р. Билс, Е. А. Карташова, Конструктивная факторизация линейных дифференциальных операторов в частных производных, ТМФ, 2005, том 145, номер 2, 165-180

DOI: https://doi.org/10.4213/tmf1892

Использование Общероссийского математического портала Math-Net.Ru подразумевает, что вы прочитали и согласны с пользовательским соглашением

http: //www . mathnet.ru/rus/agreement

Параметры загрузки:

IP : 34.227 .88 .159

26 апреля 2023 г., 15:52:57 
ТЕОРЕТИЧЕСКАЯ

И МАТЕМАТИЧЕСКАЯ

ФИЗИКА

Том 145, № 2

ноябрь, 2005

(C) 2005 г.

Р. Билс ${ }^{*}$, Е. А. Карташова ${ }^{\dagger}$

\section{КОНСТРУКТИВНАЯ ФАКТОРИЗАЦИЯ ЛИНЕЙНЫХ ДИФФЕРЕНЦИАЛЬНЫХ ОПЕРАТОРОВ В ЧАСТНЫХ ПРОИЗВОДНЫХ}

Исследуются условия, при которых линейный дифференциальный оператор в частных производных двух переменных или обыкновенный линейный дифференциальный оператор произвольного поря дка $n$ допускает факторизацию с множителем первого порядка слева. Процедура факторизации заключается в рекуррентном решении систем линейных уравнений с учетом некоторых дифференциальных условий совместности. В случае дифференциальных операторов в частных производных общего положения нет необходимости решать дифференциальное уравнение. В частных вырожденных случаях, таких как обыкновенный дифференциальный оператор, задача сводится в конечном счете к решению некоторого уравнения (некоторых уравнений) Риккати. Условия факторизации даны в явном виде для случаев второго и третьего порядков, а для случаев более высокого порядка дана схема их построения.

Ключевые слова: дифференциальный оператор, факторизация дифференциальных операторов, алгебраическая факторизация.

\section{1. ВВЕДЕНИЕ}

Факторизация линейных дифференциальных операторов (ЛДО) представляет собой очень хорошо изученную задачу, в которой доказано много строгих теорем существования (см., например, [1]). Случай линейных обыкновенных дифференциальных операторов (ЛОДО) изучен более подробно; известны разные алгоритмы факторизации для ЛОДО над различными дифференшиальными полями (см., например, работы [2]). Вероятно, первый алгоритм для случая простейшего возможного дифференциального поля (поля рациональных функций) описан в работе [3]. Известно, что в этом случае разложение на простые множители единственно [4], и задача сводится в конечном счете к решению уравнения Риккати.

Гораздо меньше известно относительно задачи факторизации ЛДО в частных производных, которая представляется значительно более сложной, чем задача для ЛОДО. В случае нескольких переменных наивное определение факторизации как представления данного оператора $n$-го порядка в виде произведения операторов более низкого по-

*Yale University, New Haven, CT, USA. E-mail: richard.beals@yale.edu

${ }^{\dagger}$ J. Kepler University, Linz, Austria. E-mail: lena@risc.uni-linz.ac.at 
рядка не обеспечивает требующего более тонкого рассмотрения свойства единственности. Сказанное иллюстрируется примером Е. Ландау [5] (сформулированным для левого произведения):

$$
\left(\partial_{x}+1\right)\left(\partial_{x}+1\right)\left(\partial_{x}+x \partial_{y}\right)=\left[\partial_{x}^{2}+x \partial_{x} \partial_{y}+\partial_{x}+(2+x) \partial_{y}\right]\left(\partial_{x}+1\right) .
$$

Тем не менее в этом направлении также были получены некоторые интересные результаты; недавно Григорьев и Шварц [6] дали алгоритм для факторизации ЛДО в частных производных с сепарабильным символом. Некоторые обобшенные определения факторизации дали начало другим алгоритмам факторизации для разложения на множители ЛДО в частных производных [7] и систем линейных дифференциальных уравнений в частных производных с рациональными функциями в качестве коэффициентов [8].

По-видимому, обшее мнение заключается в том, что наивная факторизация - даже для дифференциальных операторов двух переменных второго и третьего порядков - требует "решения уравнения Риккати в частных производных, что, в свою очередь, требует решения обыкновенного дифференциального уравнения (ОДУ) первого порядка обшего вида и, возможно, обыкновенного уравнения Риккати. Узким местом при разработке алгоритмов факторизации для ЛДО в частных производных является ОДУ первого порядка обшего вида, которое делает задачу в целом труднорешаемой, поскольку в настоящее время, вообще говоря, не существует алгоритмов решения такого уравнения" [6].

Мы считаем возможным построение явного алгоритма, который может использоваться для абсолютного разложения на линейные множители ЛДО в частных производных двух переменных произвольного порядка $n$. Слово “абсолютный" означает, что мы не фиксируем с самого начала поле коэффициентов, и наше единственное требование на коэффициенты состоит в том, что они должны быть гладкими, т.е. принадлежать некоторому подходящему дифференциальному полю. Предлагаемая здесь процедура заключается в нахождении (когда это возможно) левы $x$ множителей первого порядка, в отличие от использования правой факторизации, получившей распространение в работах нескольких последних десятилетий. Конечно, существование некоторого правого множителя для ЛДО в частных производных эквивалентно сушествованию соответствующего левого множителя для соответствующего транспонированного оператора, так что в принципе при рассмотрении левой факторизации мы ничего не теряем. Более того, операция транспонирования алгебраически тривиальна, так что мы ничего не теряем и с точки зрения вычислительного алгоритма.

Использование левой факторизации сильно упрощает все необходимые алгебраические вычисления, а также дает явные условия на коэффициенты оператора, необходимые и достаточные для сушествования множителя разложения. Простой вид этих условий позволяет увидеть принципиальное различие между факторизацией Л ДО в частных производных и ЛОДО: в первом случае задача факторизации может быть решена с помощью чисто алгебраических методов в обшем случае, а ЛОДО дает один из примеров различных вырожденных случаев, когда для факторизации в пространстве двух переменных необходимо решать уравнение Риккати.

В разделе 2 представлена общая концепция факторизации (в общем случае) посредством чисто алгебраических методов и выписаны явные условия факторизации для ЛДО 
в частных производных второго порядка. Также показано, каким образом возможность чисто алгебраической факторизации ЛДО в частных производных не вступает в противоречие с необходимостью решать уравнение Риккати для факторизации ЛОДо. Аналогичные результаты для ЛДО в частных производных и ЛОДО третьего порядка представлены в разделе 3 , а в разделе 4 представлена общая процедура факторизации для ЛДО в частных производных произвольного порядка $n$. В разделе 5 построен ряд интересных примеров, а раздел 6 содержит краткое заключительное обсуждение.

\section{2. ЛДО В ЧАСТНЫХ ПРОИЗВОДНЫХ ДВУХ ПЕРЕМЕННЫХ ВТОРОГО ПОРЯДКА}

Рассмотрим оператор

$$
A_{2}=\sum_{j+k \leqslant 2} a_{j k} \partial_{x}^{j} \partial_{y}^{k}=a_{20} \partial_{x}^{2}+a_{11} \partial_{x} \partial_{y}+a_{02} \partial_{y}^{2}+a_{10} \partial_{x}+a_{01} \partial_{y}+a_{00}
$$

с гладкими коэффициентами и будем искать факторизацию в виде

$$
A_{2}=\left(p_{1} \partial_{x}+p_{2} \partial_{y}+p_{3}\right)\left(p_{4} \partial_{x}+p_{5} \partial_{y}+p_{6}\right)
$$

Выпишем явно уравнения для величин $p_{i}$, имея в виду правило левой композиции, т.е. $\partial_{x}\left(\alpha \partial_{y}\right)=\partial_{x}(\alpha) \partial_{y}+\alpha \partial_{x y}$. Тогда во всех случаях

$$
\begin{aligned}
& a_{20}=p_{1} p_{4}, \\
& a_{11}=p_{2} p_{4}+p_{1} p_{5}, \\
& a_{02}=p_{2} p_{5}, \\
& a_{10}=\mathcal{L}\left(p_{4}\right)+p_{3} p_{4}+p_{1} p_{6}, \\
& a_{01}=\mathcal{L}\left(p_{5}\right)+p_{3} p_{5}+p_{2} p_{6}, \\
& a_{00}=\mathcal{L}\left(p_{6}\right)+p_{3} p_{6},
\end{aligned}
$$

где мы используем обозначение $\mathcal{L}=p_{1} \partial_{x}+p_{2} \partial_{y}$.

Начнем с некоторых предварительных замечаний. Задача факторизации должна рассматриваться как в некотором роде локальная задача; например, если некоторый коэффищиент не обращается в нуль тождественно, может возникнуть желание ограничиться той областью, где этот коэффициент не имеет нулей. Разумеется, поскольку полученные формулы являются аналитическими функциями данных, они могут быть глобально продолжены, если данные также являются аналитическими. Имея в виду это обстоятельство, заметим следуюшее. Если оператор не является (глобально) оператором первого порядка, то, сделав при необходимости линейную замену переменных, можно предполагать, что $a_{20} \neq 0$. В таком случае непременно выполнено условие $p_{1} \neq 0$, и без потери обшности можно считать, что $p_{1}=1$. Тогда первые три уравнения системы $(2)$, характеризуюшие члены наиболее высокого порядка, суть уравнения на переменные $p_{2}$, $p_{4}, p_{5}$, и чтобы их определить, нужно найти корень $\omega$ квадратного полинома. Вычислив $p_{2}, p_{4}, p_{5}$, можно подставить их в следующие два уравнения системы $(2)$ и получить линейную систему уравнений относительно двух переменных $p_{3}, p_{6}$, которая легко может 
быть решена, если $\omega$ - простой корень. Наконец, последнее уравнение системы (2) даст нам условие факторизации.

Действительно, на первом шаге из уравнений

$$
\begin{aligned}
& a_{20}=p_{4}, \\
& a_{11}=p_{2} p_{4}+p_{5}, \\
& a_{02}=p_{2} p_{5}
\end{aligned}
$$

следует, что

$$
\mathcal{P}_{2}\left(-p_{2}\right):=a_{20}\left(-p_{2}\right)^{2}+a_{11}\left(-p_{2}\right)+a_{02}=0 .
$$

Выбор разных корней характеристического полинома $\mathcal{P}_{2}$ приводит к разным возможным факторизациям исходного оператора (как мы увидим ниже, такая факторизация может и не сушествовать или может существовать факторизация, отвечающая одному выбору корня, и не существовать факторизации, отвечающей другому выбору). Пусть $\omega$ - корень полинома $\mathcal{P}_{2} ;$ возьмем

$$
p_{2}=-\omega, \quad \mathcal{L}=\partial_{x}-\omega \partial_{y} .
$$

Это приводит к линейной системе относительно переменных $p_{4}, p_{5} \mathrm{c} \omega$ в качестве параметра:

$$
\begin{gathered}
p_{4}=a_{20}, \\
-\omega p_{4}+p_{5}=a_{11},
\end{gathered}
$$

или в матричном виде

$$
\left(\begin{array}{cc}
1 & 0 \\
-\omega & 1
\end{array}\right)\left(\begin{array}{l}
p_{4} \\
p_{5}
\end{array}\right)=\left(\begin{array}{l}
a_{20} \\
a_{11}
\end{array}\right), \quad\left(\begin{array}{l}
p_{4} \\
p_{5}
\end{array}\right)=\left(\begin{array}{ll}
1 & 0 \\
\omega & 1
\end{array}\right)\left(\begin{array}{l}
a_{20} \\
a_{11}
\end{array}\right) .
$$

Таким образом,

$$
p_{1}=1, \quad p_{2}=-\omega, \quad p_{4}=a_{20}, \quad p_{5}=a_{20} \omega+a_{11} .
$$

На втором шаге из четвертого и пятого уравнений системы (2) и формул (3) получаем

$$
\begin{aligned}
& a_{10}=\mathcal{L} a_{20}+p_{3} a_{20}+p_{6}, \\
& a_{01}=\mathcal{L}\left(a_{11}+a_{20} \omega\right)+p_{3}\left(a_{11}+a_{20} \omega\right)-\omega p_{6} .
\end{aligned}
$$

Если корень $\omega$ простой, т.е. $\mathcal{P}_{2}^{\prime}(\omega)=2 a_{20} \omega+a_{11} \neq 0$, то эти уравнения имеют единственное решение

$$
\begin{aligned}
& p_{3}=\frac{\omega a_{10}+a_{01}-\omega \mathcal{L} a_{20}-\mathcal{L}\left(a_{20} \omega+a_{11}\right)}{2 a_{20} \omega+a_{11}}, \\
& p_{6}=\frac{\left(a_{20} \omega+a_{11}\right)\left(a_{10}-\mathcal{L} a_{20}\right)-a_{20}\left(a_{01}-\mathcal{L}\left(a_{20} \omega+a_{11}\right)\right)}{2 a_{20} \omega+a_{11}} .
\end{aligned}
$$

Таким образом, все коэффициенты $p_{1}, p_{2}, \ldots, p_{6}$ вычислены. 
На третьем шаге из последнего уравнения системы (2) может быть явно выписано соответствуюшее условие факторизации

$$
\begin{aligned}
a_{00}= & \mathcal{L}\left\{\frac{\omega a_{10}+a_{01}-\mathcal{L}\left(2 a_{20} \omega+a_{11}\right)}{2 a_{20} \omega+a_{11}}\right\}+\frac{\omega a_{10}+a_{01}-\mathcal{L}\left(2 a_{20} \omega+a_{11}\right)}{2 a_{20} \omega+a_{11}} \times \\
& \times \frac{a_{20}\left(a_{01}-\mathcal{L}\left(a_{20} \omega+a_{11}\right)\right)+\left(a_{20} \omega+a_{11}\right)\left(a_{10}-\mathcal{L} a_{20}\right)}{2 a_{20} \omega+a_{11}}
\end{aligned}
$$

и остается только проверить его. Таким образом, факторизация для двух переменньх, ког да она возможна, может (как правило) быть достигнута чисто алгебраическими средствами.

ЗАМЕчАниЕ. В ходе представленного выше рассмотрения мы предполагали, что $\omega$ является простым корнем, так что $\left(a_{11}+2 a_{20} \omega\right) \neq 0$. Предположим теперь, что это условие нарушено тождественно в некоторой области. Тогда, исключая коэффициент $p_{6}$ из системы (4), мы получаем необходимое условие

$$
a_{10} \omega+a_{01}-\mathcal{L}\left(a_{20} \omega+a_{11}\right)-\omega \mathcal{L} a_{20}=0 .
$$

Если это условие выполняется, то можно использовать первое уравнение системы (4) для нахождения $p_{6}$ из $p_{3}$. В таком случае последнее уравнение системы $(2)$ дает уравнение Риккати для коэффициента $p_{3}$

Рассмотрим подробно случай обыкновенного дифференциального оператора второго порядка как частный случай нашего исходного ЛДО в частных производных. В этом случае $\omega$ - двукратный корень характеристического уравнения, и необходимое условие (5) непременно выполнено. В действительности система (2) принимает вид

$$
\begin{aligned}
& a_{20}=p_{4}, \\
& a_{10}=\mathcal{L}\left(p_{4}\right)+p_{3} p_{4}+p_{6}, \\
& a_{00}=\mathcal{L}\left(p_{6}\right)+p_{3} p_{6},
\end{aligned}
$$

т.е. мы имеем только три нетривиальных уравнения. Первое уравнение снова показывает, что $p_{4}=a_{20}$, член $\mathcal{L}\left(p_{4}\right)=\dot{a}_{20}$ известен, а коэффициент $p_{6}$ может быть явно выписан в виде $p_{6}=\left(a_{10}-\dot{a}_{20}-a_{20}\right) p_{3}$. Подстановка выражения для $p_{4}$ в уравнение для $a_{00}$ немедленно дает уравнение

$$
a_{00}=\dot{a}_{10}-\ddot{a}_{20}-a_{20} \dot{p}_{3}-\dot{a}_{20} p_{3}+\left(a_{10}-\dot{a}_{20}-a_{20} p_{3}\right) p_{3},
$$

которое есть уравнение Риккати

$$
\dot{\psi}+\psi^{2}+\frac{2 \dot{a}_{20}-a_{10}}{a_{20}} \psi+\frac{a_{00}+\ddot{a}_{20}-\dot{a}_{10}}{a_{20}}=0, \quad p_{3}=\psi .
$$

Для того чтобы иметь возможность найти общее решение в явном виде, нужно знать по крайней мере одно из решений этого уравнения. Решение, конечно, будет зависеть от точного вида коэффициентов $a_{i j}=a_{i j}(x)$. 


\section{3. ЛДО В ЧАСТНЫХ ПРОИЗВОДНЫХ ТРЕТЬЕГО ПОРЯДКА}

Рассмотрим теперь оператор

$$
\begin{aligned}
A_{3}= & \sum_{j+k \leqslant 3} a_{j k} \partial_{x}^{j} \partial_{y}^{k}=a_{30} \partial_{x}^{3}+a_{21} \partial_{x}^{2} \partial_{y}+a_{12} \partial_{x} \partial_{y}^{2}+a_{03} \partial y^{3}+ \\
& +a_{20} \partial_{x}^{2}+a_{11} \partial_{x} \partial_{y}+a_{02} \partial_{y}^{2}+a_{10} \partial_{x}+a_{01} \partial_{y}+a_{00}
\end{aligned}
$$

с гладкими коэффициентами и будем искать факторизацию в виде

$$
A_{3}=\left(p_{1} \partial_{x}+p_{2} \partial_{y}+p_{3}\right)\left(p_{4} \partial_{x}^{2}+p_{5} \partial_{x} \partial_{y}+p_{6} \partial_{y}^{2}+p_{7} \partial_{x}+p_{8} \partial_{y}+p_{9}\right) .
$$

Условия факторизации описьваются следуюшей системой:

$$
\begin{aligned}
& a_{30}=p_{1} p_{4}, \\
& a_{21}=p_{2} p_{4}+p_{1} p_{5}, \\
& a_{12}=p_{2} p_{5}+p_{1} p_{6}, \\
& a_{03}=p_{2} p_{6}, \\
& a_{20}=\mathcal{L}\left(p_{4}\right)+p_{3} p_{4}+p_{1} p_{7}, \\
& a_{11}=\mathcal{L}\left(p_{5}\right)+p_{3} p_{5}+p_{2} p_{7}+p_{1} p_{8}, \\
& a_{02}=\mathcal{L}\left(p_{6}\right)+p_{3} p_{6}+p_{2} p_{8}, \\
& a_{10}=\mathcal{L}\left(p_{7}\right)+p_{3} p_{7}+p_{1} p_{9}, \\
& a_{01}=\mathcal{L}\left(p_{8}\right)+p_{3} p_{8}+p_{2} p_{9}, \\
& a_{00}=\mathcal{L}\left(p_{9}\right)+p_{3} p_{9},
\end{aligned}
$$

где $\mathcal{L}=p_{1} \partial_{x}+p_{2} \partial_{y}$.

Снова без потери обшности можно предполагать, что коэффициент при члене наиболее высокого порядка по $\partial_{x}$ не обращается в нуль, а линейный множитель нормирован:

$$
a_{30} \neq 0, \quad p_{1}=1 \text {. }
$$

Первые четыре уравнения системы (9), описывающие члены наиболее высокого порядка, суть уравнения относительно переменных $p_{2}, p_{4}, p_{5}, p_{6}$. Решение этих уравнений требует выбора корня $-p_{2}$ некоторого полинома третьей степени. Как только такой выбор сделан, оставшиеся коэффициенты старшего порядка $p_{4}, p_{5}, p_{6}$ легко находятся. Коэффициенты старшего порядка могут теперь быть подставлены в следующие четыре уравнения системы (9). Первые три из этих четырех уравнений теперь представляют собой линейную систему уравнений относительно переменных $p_{3}, p_{7}, p_{8}$, которая легко решается. Следуюшее уравнение линейно относительно переменной $p_{9}$; это означает, что все переменные $p_{i}, i=1,2, \ldots, 9$, найдены. Последние два уравнения системы (9) дадут тогда условия факторизации.

А именно, на первом шаге из уравнений

$$
\begin{aligned}
& a_{30}=p_{4}, \\
& a_{21}=p_{2} p_{4}+p_{5}, \\
& a_{12}=p_{2} p_{5}+p_{6}, \\
& a_{03}=p_{2} p_{6}
\end{aligned}
$$


следует, что

$$
\mathcal{P}_{3}\left(-p_{2}\right):=a_{30}\left(-p_{2}\right)^{3}+a_{21}\left(-p_{2}\right)^{2}+a_{12}\left(-p_{2}\right)+a_{03}=0 .
$$

Как и в случае второго порядка, выбирая $p_{2}=-\omega$, где $\omega$-корень характеристического полинома $\mathcal{P}_{3}$, мы получаем линейную систему относительно коэффициентов $p_{4}, p_{5}, p_{6}$ с $\omega$ в качестве параметра. Тогда снова

$$
p_{2}=-\omega, \quad \mathcal{L}=\partial_{x}-\omega \partial_{y},
$$

что дает

$$
\begin{aligned}
& a_{30}=p_{4}, \\
& a_{21}=-\omega p_{4}+p_{5}, \\
& a_{12}=-\omega p_{5}+p_{6},
\end{aligned}
$$

т.е.

$$
\left(\begin{array}{ccc}
1 & 0 & 0 \\
-\omega & 1 & 0 \\
0 & -\omega & 1
\end{array}\right)\left(\begin{array}{l}
p_{4} \\
p_{5} \\
p_{6}
\end{array}\right)=\left(\begin{array}{l}
a_{30} \\
a_{21} \\
a_{12}
\end{array}\right), \quad\left(\begin{array}{l}
p_{4} \\
p_{5} \\
p_{6}
\end{array}\right)=\left(\begin{array}{ccc}
1 & 0 & 0 \\
\omega & 1 & 0 \\
\omega^{2} & \omega & 1
\end{array}\right)\left(\begin{array}{l}
a_{30} \\
a_{21} \\
a_{12}
\end{array}\right)
$$

Таким образом,

$$
\begin{aligned}
& p_{1}=1, \quad p_{2}=-\omega, \quad p_{4}=a_{30}, \\
& p_{5}=a_{30} \omega+a_{21}, \quad p_{6}=a_{30} \omega^{2}+a_{21} \omega+a_{12} .
\end{aligned}
$$

На втором шаге из уравнений

$$
\begin{aligned}
& a_{20}=\mathcal{L}\left(p_{4}\right)+p_{3} p_{4}+p_{1} p_{7} \\
& a_{11}=\mathcal{L}\left(p_{5}\right)+p_{3} p_{5}+p_{2} p_{7}+p_{1} p_{8} \\
& a_{02}=\mathcal{L}\left(p_{6}\right)+p_{3} p_{6}+p_{2} p_{8}
\end{aligned}
$$

и формул (10) получаем

$$
\begin{aligned}
a_{20}-\mathcal{L} a_{30} & =p_{3} a_{30}+p_{7}, \\
a_{11}-\mathcal{L}\left(a_{30} \omega+a_{21}\right) & =p_{3}\left(a_{30} \omega+a_{21}\right)-\omega p_{7}+p_{8}, \\
a_{02}-\mathcal{L}\left(a_{30} \omega^{2}+a_{21} \omega+a_{12}\right) & =p_{3}\left(a_{30} \omega^{2}+a_{21} \omega+a_{12}\right)-\omega p_{8} .
\end{aligned}
$$

Как линейная система для определения $p_{3}, p_{7}, p_{8}$, данная система имеет детерминант

$$
3 a_{30} \omega^{2}+2 a_{21} \omega+a_{12}=\mathcal{P}^{\prime}(\omega)
$$

Таким образом, если $\omega-$ простой корень, то эта система имеет единственное решение

$$
\begin{aligned}
p_{3} & =\frac{\omega^{2}\left(a_{20}-\mathcal{L} a_{30}\right)+\omega\left(a_{11}-\mathcal{L}\left(a_{30} \omega+a_{21}+a_{02}\right)\right)-\mathcal{L}\left(a_{30} \omega^{2}+a_{21} \omega+a_{12}\right)}{3 a_{30} \omega^{2}+2 a_{21} \omega+a_{12}}, \\
p_{7} & =\frac{a_{20}-\mathcal{L} a_{30}}{3 a_{30} \omega^{2}+2 a_{21} \omega+a_{12}}-\frac{a_{30}}{3 a_{30} \omega^{2}+2 a_{21} \omega+a_{12}} p_{3}, \\
p_{8} & =\frac{\omega\left(a_{20}-\mathcal{L} a_{30}\right)+a_{11}-\mathcal{L}\left(a_{30} \omega+a_{21}\right)}{3 a_{30} \omega^{2}+2 a_{21} \omega+a_{12}}-\frac{a_{30} \omega+a_{21}}{3 a_{30} \omega^{2}+2 a_{21} \omega+a_{12}} p_{3} .
\end{aligned}
$$


Для того чтобы найти последний коэффициент $p_{9}$, используем восьмое уравнение системы $(9)$.

Теперь все коэффициенты $p_{i}, i=1,2, \ldots, 9$, вычислены в предположении, что $\omega-$ простой корень.

На третьем шаге из двух последних уравнений системы (9) могут быть выписаны все необходимые условия факторизации. Здесь мы не будем делать этого, поскольку соответствуюшие формулы достаточно громоздки и ничего не прибавляют для понимания основной идеи. Если условия выполняются, то явные формулы факторизации могут быть выписаны, как и для оператора второго порядка. Отличие состоит в том, что в рассматриваемом случае определяемый членами наиболее высокого порядка полином является полиномом третьей степени, и мы имеем не одно, а два условия факторизации.

Рассматривая теперь ЛОДО как частный случай ЛДО в частных производных, перепишем систему (9) с $p_{1}=1$ в виде

$$
\begin{aligned}
& a_{30}=p_{4}, \\
& a_{20}=p_{3} p_{4}+p_{1} p_{7}+\dot{p}_{4}, \\
& a_{10}=p_{3} p_{7}+p_{9}+\dot{p}_{7}, \\
& a_{00}=p_{3} p_{9}+\dot{p}_{9} .
\end{aligned}
$$

Как и в случае оператора второго порядка, $\omega=0$ есть кратный корень полинома $\mathcal{P}_{3}$. После исключения $p_{4}=a_{30}$ и решения второго и третьего уравнений соответственно для $p_{7}$ и $p_{9}$ в терминах $p_{3}$ мы видим, что задача факторизации для ЛОДО третьего порядка эквивалентна следующей системе нелинейных ОДУ первого порядка относительно единственной неизвестной функции $p_{3}$ :

$$
\begin{aligned}
& a_{10}=\frac{p_{7}\left(a_{20}-\dot{a}_{30}-p_{7}\right)}{a_{30}}+p_{9}+\dot{p}_{7}, \\
& a_{00}=\frac{p_{9}\left(a_{20}-\dot{a}_{30}-p_{7}\right)}{a_{30}}+\dot{p}_{9},
\end{aligned}
$$

или, что то же самое, некоторому нелинейному ОДУ второго порядка.

\section{4. ЛДО В ЧАСТНЫХ ПРОИЗВОДНЫХ ПРОИЗВОЛЬНОГО ПОРЯДКА}

Рассмотрим теперь задачу факторизации для ЛДО в частных производных порядка $n$ обшего вида

$$
A_{n}=\sum_{j+k \leqslant n} a_{j k} \partial_{x}^{j} \partial_{y}^{k}=\left(p_{1} \partial_{x}+p_{2} \partial_{y}+p_{3}\right)\left(\sum_{j+k<n} p_{j k} \partial_{x}^{k} \partial_{y}^{j}\right)
$$

и используем те же предположения и обозначения, что и выше. А именно, некоторые из $a_{j k}, j+k=n$, не равны нулю, так что можно:

1) предполагать, что после линейной замены переменных $a_{n 0} \neq 0$;

2) положить $p_{1}=1$ и $p_{j k}=0$, если $j<0$ или $k<0$;

3 ) обозначить $p_{2}$ как $-\omega$ и $\mathcal{L}=p_{1} \partial_{x}+p_{2} \partial_{y}=\partial_{x}-\omega \partial_{y}$. 
Тогда уравнения, описываюшие члены наиболее высокого порядка, принимают вид $p_{1} p_{j-1, k}+p_{2} p_{j, k-1}=a_{j k}$, т.e.

$$
p_{j-1, k}-\omega p_{j, k-1}=a_{j k}, \quad j+k=n,
$$

в то время как уравнения более низких порядков принимают вид

$$
\mathcal{L} p_{j k}+p_{3} p_{j k}+p_{j-1, k}-\omega p_{j, k-1}=a_{j k}, \quad j+k<n .
$$

Имея в виду, что $j=n-k$ для $n$ первых уравнений (14), перепишем их как

$$
p_{n-1-k, k}-\omega p_{n-k, k-1}=a_{n-k, k}, \quad k=0,1, \ldots, n-1,
$$

или в матричном виде

$$
\left(\begin{array}{cccccc}
1 & 0 & 0 & \ldots & 0 & 0 \\
-\omega & 1 & 0 & \ldots & 0 & 0 \\
0 & -\omega & 1 & \ldots & 0 & 0 \\
\ldots \ldots & \ldots & \ldots & \ldots & \ldots \\
0 & 0 & 0 & \ldots & -\omega & 1
\end{array}\right)\left(\begin{array}{c}
p_{n-1,0} \\
p_{n-2,1} \\
p_{n-3,2} \\
\ldots \ldots \\
p_{0, n-1}
\end{array}\right)=\left(\begin{array}{c}
a_{n, 0} \\
a_{n-1,1} \\
a_{n-2,2} \\
\ldots \ldots \\
a_{1, n-1}
\end{array}\right)
$$

Если корень $\omega$ известен, то единственное решение может быть выписано в виде

$$
\left(\begin{array}{l}
p_{n-1,0} \\
p_{n-2,1} \\
p_{n-3,2} \\
\ldots \ldots \\
p_{0, n-1}
\end{array}\right)=\left(\begin{array}{cccccc}
1 & 0 & 0 & \ldots & 0 & 0 \\
\omega & 1 & 0 & \ldots & 0 & 0 \\
\omega^{2} & \omega & 1 & \ldots & 0 & 0 \\
\ldots \ldots & \ldots & \ldots & \ldots \ldots & \ldots \\
\omega^{n-1} & \omega^{n-2} & \omega^{n-3} & \ldots & \omega & 1
\end{array}\right)\left(\begin{array}{c}
a_{n, 0} \\
a_{n-1,1} \\
a_{n-2,2} \\
\ldots \ldots \\
a_{1, n-1}
\end{array}\right)
$$

так что

$$
p_{n-k, k}=a_{n, 0} \omega^{k}+a_{n-1,1} \omega^{k-1}+\cdots+a_{n-k, k} .
$$

Уравнение (14) с $k=n$, с учетом равенства $-\omega p_{0, n-1}=a_{0, n}$, дает окончательно

$$
\mathcal{P}_{n}(\omega):=a_{n, 0} \omega^{n}+a_{n-1,1} \omega^{n-1}+\cdots+a_{1, n-1} \omega+a_{0, n}=0 .
$$

Выбирая в качестве $\omega$ любой из корней этого полинома, мы затем однозначно определяем коэффициенты $p_{j, k}$ для всех $j+k=n$.

Рассмотрим теперь $n$ уравнений системы (15), соответствующих членам $(n-1)$-го порядка, т.е. случаю $j+k=n-1$ :

$$
p_{n-2-k, k}-\omega p_{n-k-1, k-1}=b_{n-1-k, k}-p_{3} p_{n-1-k, k},
$$

где функции $p_{n-1-k, k}$ и члены

$$
b_{n-1-k, k}=a_{n-1-k, k}-\mathcal{L} p_{n-1-k, k}
$$


уже известны. В матричном виде эти уравнения суть

$$
\left(\begin{array}{ccccccc}
p_{n-1,0} & 1 & 0 & 0 & \ldots & 0 & 0 \\
p_{n-2,1} & -\omega & 1 & 0 & \ldots & 0 & 0 \\
p_{n-3,2} & 0 & -\omega & 1 & \ldots & 0 & 0 \\
\ldots \ldots & \ldots & \ldots & \ldots & \ldots & \ldots & \ldots \\
p_{0, n-1} & 0 & 0 & 0 & \ldots & 0 & -\omega
\end{array}\right)\left(\begin{array}{c}
p_{3} \\
p_{n-2,0} \\
p_{n-3,1} \\
\ldots \ldots \\
p_{0, n-2}
\end{array}\right)=\left(\begin{array}{c}
b_{n-1,0} \\
b_{n-2,1} \\
b_{n-3,2} \\
\ldots \ldots \\
b_{0, n-1}
\end{array}\right)
$$

По индукции детерминант матрицы такого вида равен

$$
(-1)^{n-1}\left[p_{n-1,0} \omega^{n-1}+p_{n-2,1} \omega^{n-2}+\cdots+p_{0, n-1}\right] .
$$

Из уравнений (17) следует, что член в квадратных скобках равен

$$
n a_{n, 0} \omega^{n-1}+(n-1) a_{n-2,1} \omega^{n-2}+\cdots+a_{1, n-1}=\mathcal{P}_{n}^{\prime}(\omega) .
$$

Поэтому если $\omega-$ простой корень, то уравнения (18) для коэффициентов $p_{3}$ и $p_{n-2-k, k}$ имеют единственное решение. Умножая $j$-е уравнение системы на $\omega^{n-j}$ и складьвая, находим

$$
\mathcal{P}_{n}^{\prime}(\omega) p_{3}=b_{n-1,0} \omega^{n-1}+b_{n-2,1} \omega^{n-2}+\cdots+b_{0, n-1} .
$$

Если $\omega-$ простой корень, то это уравнение определяет $p_{3}$, и можно определить $p_{n-2-k, k}$, перенося члены, содержашие $p_{3}$, в первых $n-1$ уравнениях рассматриваемой системы в правую часть, так что решение для коэффициентов $p_{n-2-k, k}$ в терминах коэффициентов $p_{3}$ и других известных функций есть

$$
\left(\begin{array}{c}
p_{n-2,0} \\
p_{n-3,1} \\
p_{n-4,2} \\
\ldots \ldots \\
p_{0, n-2}
\end{array}\right)=\left(\begin{array}{cccccc}
1 & 0 & 0 & \ldots & 0 & 0 \\
\omega & 1 & 0 & \ldots & 0 & 0 \\
\omega^{2} & \omega & 1 & \ldots & 0 & 0 \\
\ldots \ldots & \ldots & \ldots & \ldots \ldots & \ldots \\
\omega^{n-2} & \omega^{n-3} & \omega^{n-4} & \ldots & \omega & 1
\end{array}\right)\left(\begin{array}{c}
b_{n-1,0}-p_{3} p_{n-1,0} \\
b_{n-2,1}-p_{3} p_{n-2,1} \\
b_{n-3,2}-p_{3} p_{n-3,2} \\
\ldots \ldots \ldots \ldots \ldots \ldots \\
b_{1, n-2}-p_{3} p_{1, n-2}
\end{array}\right)
$$

как и ранее.

Продолжая считать, что $\omega$ - простой корень, продолжим данную процедуру еще на один шаг. Первые $n-2$ из $n-1$ уравнений для коэффициентов $p_{n-3-k, k}$ могут быть записаны в матричном виде, подобном (16), с известными функциями в правой части, так что решение может быть выписано, как и выше. Таким образом, мы уже определили коэффициенты $p_{j}$ первого множителя и все коэффициенты $p_{j k}, j+k \leqslant n-3$, второго множителя. Однако на этом шаге есть еще одно уравнение:

$$
-\omega p_{0, n-3}=a_{1, n-3}-\left(\mathcal{L}+p_{3}\right) p_{1, n-3}
$$

Поскольку обе части уравнения уже были определены, оно является необходимым условием сушествования факторизации при данном выборе корня $\omega$. При продолжении процедуры та же ситуация возникает на каждом шаге. Таким образом, если условие выполняется на одном шаге, можно переходить к следующему, и либо процесс окончится неудачей, либо существует ровно $n-1$ полиномиальных условий, которым удовлетворяют коэффициенты, (простой) корень $\omega$ и их производные. 
Допустим, что мы отказались от условия, что $\omega$ - простой корень, и предположим, что $\mathcal{P}_{n}^{\prime}(\omega) \equiv 0$ в некоторой области. Тогда уравнение (19) с нулевой правой частью является необходимым и достаточным условием разрешимости полной системы уравнений для коэффициентов $p_{3}$ и $p_{n-2-k, k}$. Если это условие выполнено, то можно осуществить выбор коэффициента $p_{3}$ и определить коэффициенты $p_{n-2-k, k}$ из (20). Далее мы сталкиваемся с условиями факторизации, которые могут быть выражены как полиномиальные уравнения по коэффициенту $p_{3}$ и его производным (с коэффициентами, которые являются полиномами по коэффициентам $a_{j k}$ и их производным, а также по корню $\omega$ и его производным); эти уравнения можно считать обобщенными уравнениями Риккати для $p_{3}$.

Итак, начавшись с простого корня полинома $\mathcal{P}_{n}(\omega)$ в случае обшего положения $a_{n, 0} \neq 0$, процесс факторизации продолжается алгебраически (в терминах коэффициентов $a_{j k}$, корня $\omega$ и их производных) до тех пор, пока это возможно, что определяется $n-2$ полиномиальными ограничениями. Начавшись с двукратного корня, процесс затем подчиняется одному такому полиномиальному ограничению, остальные ограничения являются $n-2$ обобшенными уравнениями Риккати для $p_{3}$.

По очевидным причинам мы называем данную процедуру исключения порядковой редукиией. В попытке представить оператор $n$-го порядка как произведение оператора первого порядка и оператора $(n-1)$-го порядка мы смотрим на уравнения, связанные с членами данной степени оператора порядка $n$, в порядке убывания. Первое множество уравнений дает члены старшего порядка обоих множителей (по модулю выбора корня характеристического уравнения), следующее множество определяет коэффициенты следуюшего более низкого порядка и т.д.

\section{5. НЕКОТОРЫЕ ПРИМЕРЫ}

\section{1. Общий ЛДО в частных производных второго порядка.}

ПРИМЕР 1. ГИПЕРБОЛИЧЕСКИЙ ОПЕРАТоР: $a_{20}=0, a_{11}=1, a_{02}=0$, т.е. $L_{2}:=$ $\partial_{x} \partial_{y}+a_{10} \partial_{x}+a_{01} \partial_{y}+a_{00}$. В этом случае возможна любая из двух различных факторизаций:

а) если $a_{00}=\partial_{x} a_{10}+a_{10} a_{01}$, то

$$
L_{2}=\left(\partial_{x}+a_{01}\right)\left(\partial_{y}+a_{10}\right),
$$

или

б) если $a_{00}=\partial_{y} a_{01}+a_{10} a_{01}$, то

$$
L_{2}=\left(\partial_{y}+a_{10}\right)\left(\partial_{x}+a_{01}\right) \text {. }
$$

Рассмотрим случай такого оператора, известный как оператор Пуассона:

$$
L_{2}=\partial_{x} \partial_{y}+\frac{1}{x+y}\left(\alpha \partial_{x}+\beta \partial_{y}\right)+\frac{\gamma}{(x+y)^{2}}
$$


где $\alpha, \beta, \gamma$ - константы. Факторизация "а" или "б" возможна, только если $\gamma=\alpha(\beta-1)$ или $\gamma=\beta(\alpha-1)$, и имеет явньй вид

$$
L_{2}=\left(\partial_{x}+\frac{\beta}{x+y}\right)\left(\partial_{y}+\frac{\alpha}{x+y}\right)
$$

или

$$
L_{2}=\left(\partial_{y}+\frac{\alpha}{x+y}\right)\left(\partial_{x}+\frac{\beta}{x+y}\right) \text {, }
$$

соответственно.

Заметим, что в частном случае $\gamma=0$ оператор Пуассона сводится к оператору, рассмотренному в примере 4 работы [6], и соответствующие результаты могут быть немедленно получены из приведенных выше формул.

ПРИМЕР 2. ПАРАБОЛИЧЕСКИЙ ОПЕРАТОР. ОПератор

$$
A_{\mathrm{p}}=a_{20} \partial_{x}^{2}+a_{11} \partial_{x} \partial_{y}+a_{02} \partial_{y}^{2}+a_{10} \partial_{x}+a_{01} \partial_{y}+a_{00}
$$

является параболическим, только если $a_{11}^{2}-4 a_{20} a_{02}=0$. Это условие означает, что характеристический полином

$$
\mathcal{P}_{2}:=a_{20} \omega^{2}+a_{11} \omega+a_{02}
$$

имеет двукратный корень $\omega=-a_{11} / a_{20}$, и из рассмотрения раздела 2 следует, что факторизация требует решения уравнения Риккати для $p_{3}$.

ПрИмЕР 3. ЭлЛИПтИчЕСКИй опЕРАТОР. Оператор

$$
A_{\mathrm{e}}=a_{20} \partial_{x}^{2}+a_{11} \partial_{x} \partial_{y}+a_{02} \partial_{y}^{2}+a_{10} \partial_{x}+a_{01} \partial_{y}+a_{00}
$$

(с вешественными коэффициентами) является по определению эллиптическим, если $a_{11}^{2}-4 a_{20} a_{02}<0$, так что корни характеристического полинома $\mathcal{P}_{2}$ комплексные. Поэтому факторизация возможна лишш над полем, содержащим $\mathbb{C}$.

5.2. Роль нулевого члена. Порядок факторизации. Рассмотрим следующие операторы двух переменных второго порядка:

$$
A_{a}=\partial_{x}^{2}-\partial_{y}^{2}+x \partial_{y}+y \partial_{x}+\frac{1}{4}\left(y^{2}-x^{2}\right)+a,
$$

где $a$ - вешественная константа.

ПримеР 4. Будем искать факторизацию в виде

$$
A_{a}=\left(p_{1} \partial_{x}+p_{2} \partial_{y}+p_{3}\right)\left(p_{4} \partial_{x}+p_{5} \partial_{y}+p_{6}\right)
$$

Равенство $p_{1} p_{4}=1$ непременно выполнено, и без существенного ограничения общности можно взять $p_{1}=p_{4}=1$. Тогда нетрудно показать, что факторизация может иметь лишь один из двух видов:

$$
A_{a}=L_{a}^{+} L_{a}^{-}=\left(\partial_{x}+\partial_{y}+b\right)\left(\partial_{x}-\partial_{y}+c\right)
$$


или

$$
A_{a}=L_{a}^{-} L_{a}^{+}=\left(\partial_{x}-\partial_{y}+b\right)\left(\partial_{x}+\partial_{y}+c\right) .
$$

Как следует из приведенных выше результатов, а также может быть проверено непосредственно, справедливы следующие утверждения.

1. Оператор

$$
A_{a}=\partial_{x}^{2}-\partial_{y}^{2}+y \partial_{x}+x \partial_{y}+\frac{1}{4}\left(y^{2}-x^{2}\right)+a
$$

не допускает факторизации ни одного из видов $(22),(23)$, за исключением случая $a=$ \pm 1 .

2. Оператор

$$
A_{1}=\partial_{x}^{2}-\partial_{y}^{2}+x \partial_{y}+y \partial_{x}+\frac{1}{4}\left(y^{2}-x^{2}\right)+1
$$

допускает факторизацию вида (22), но не допускает факторизации вида (23).

3. Оператор

$$
A_{-1}=\partial_{x}^{2}-\partial_{y}^{2}+x \partial_{y}+y \partial_{x}+\frac{1}{4}\left(y^{2}-x^{2}\right)-1
$$

допускает факторизацию вида (23), но не допускает факторизации вида (22).

Действительно,

$$
A_{1}=\left[\partial_{x}+\partial_{y}+\frac{1}{2}(y-x)\right]\left[\partial_{x}-\partial_{y}+\frac{1}{2}(y+x)\right]
$$

и

$$
A_{-1}=\left[\partial_{x}-\partial_{y}+\frac{1}{2}(y+x)\right]\left[\partial_{x}+\partial_{y}+\frac{1}{2}(y-x)\right] .
$$

Этот пример показывает, что существование факторизации критическим образом зависит от члена нулевого порядка, а также что оператор может иметь левый фактор некоторого вида, но не иметь правого фактора того же вида, и обратно.

Мы показали, что в случае двух переменных вопрос о сушествовании левого фактора первого порядка заданного вида может быть решен, а сам фактор вычислен систематическим образом с использованием простых и (в общем случае) чисто алгебраических процедур.

ПримеР 5. Вопрос о сушествовании правого фактора первого порядка может быть сведен к аналогичному вопросу для левого фактора посредством формального транспонирования. В качестве примера найдем таким образом правый фактор для оператора $A_{1}$. Чтобы воспользоваться тем, что уже известно, сделаем замену координат $y \rightarrow-y$, при которой оператор $A_{1}$ преобразуется к виду

$$
\tilde{A}_{1}=\partial_{x}^{2}-\partial_{y}^{2}-y \partial_{x}-x \partial_{y}+\frac{1}{4}\left(y^{2}-x^{2}\right)+1 .
$$

Транспонированньй оператор имеет вид

$$
\tilde{A}_{1}^{\mathrm{T}}=\partial_{x}^{2}-\partial_{y}^{2}+y \partial_{x}+x \partial_{y}+\frac{1}{4}\left(y^{2}-x^{2}\right)+1=A_{1}=L_{1}^{+}\left(L_{1}^{-}\right) .
$$

2 Теоретическая и математическая физика, т. 145, № 2, 2005 г. 
Поэтому

$$
\tilde{A}_{1}=\left(L_{1}^{-}\right)^{\mathrm{T}}\left(L_{1}^{+}\right)^{\mathrm{T}}=\left(-L_{1}^{-}\right)^{\mathrm{T}}\left(-L_{1}^{+}\right)^{\mathrm{T}} .
$$

Теперь согласно (24)

$$
\left(-L_{1}^{+}\right)^{\mathrm{T}}=-\left[\partial_{x}+\partial_{y}+\frac{1}{2}(y-x)\right]^{\mathrm{T}}=\partial_{x}+\partial_{y}-\frac{1}{2}(y-x) .
$$

Обратная замена координат $y \rightarrow-y$ преобразует последний оператор к виду $\partial_{x}-\partial_{y}+$ $(y+x) / 2$. В соответствии с (25) этот оператор является правым фактором для оператора $A_{1}$, согласованным с (24).

5.3. Классы факторизуемых ЛДО в частных производных. Налагая некоторые ограничения на $a_{i j}$ как функции $x$ и $y$, можно описать все операторы, допускаюшие факторизацию в заданном классе функций. Представим явные факторизации для одного случая гиперболических операторов:

$$
A_{2}:=\partial_{x x}-\partial_{y y}+a_{10} \partial_{x}+a_{01} \partial_{y}+a_{00}
$$

т.е. $a_{20}=1, a_{11}=0, a_{02}=-1$. В этом случае возможны только две факторизации.

СлучАй 1 . Если имеет место равенство $4 a_{00}=2\left(\partial_{x}+\partial_{y}\right)\left(a_{10}+a_{01}\right)+\left(a_{10}^{2}-a_{01}^{2}\right)$, то мы имеем следуюшую факторизацию:

$$
A_{2}=\left(\partial_{x}+\partial_{y}+\frac{a_{10}-a_{01}}{2}\right)\left(\partial_{x}-\partial_{y}+\frac{a_{10}+a_{01}}{2}\right) .
$$

СлучАй 2. Если имеет место равенство $4 a_{00}=2\left(\partial_{x}+\partial_{y}\right)\left(a_{10}-a_{01}\right)+\left(a_{10}^{2}-a_{01}^{2}\right)$, то мы имеем следуюшую факторизацию:

$$
A_{2}=\left(\partial_{x}+\partial_{y}+\frac{a_{10}+a_{01}}{2}\right)\left(\partial_{x}-\partial_{y}+\frac{a_{10}-a_{01}}{2}\right) .
$$

В качестве простого примера рассмотрим случай, когда коэффициенты низких порядков $a_{10}, a_{01}, a_{00}$ являются линейными функциями одной переменной $x$, т.е.

$$
\begin{aligned}
& a_{10}=b_{10,1} x+b_{10,0}, \\
& a_{01}=b_{01,1} x+b_{01,0}, \\
& a_{00}=b_{00,1} x+b_{00,0} .
\end{aligned}
$$

Тогда условие факторизации (27) есть

$$
\begin{aligned}
4 b_{00,1} x+4 b_{00,0}= & 2 b_{10,1}+2 b_{01,1}+b_{10,1}^{2} x^{2}+ \\
& +2 b_{10,1} b_{10,0} x+b_{10,0}^{2}-b_{01,1}^{2} x^{2}-2 b_{01,1} b_{01,0} x-b_{01,0}^{2},
\end{aligned}
$$

что приводит к члену нулевого порядка

$$
4 b_{00,0}=2 b_{10,1}+2 b_{01,1}+b_{10,0}^{2}-b_{01,0}^{2},
$$


линейному члену

$$
4 b_{00,1}=2 b_{10,1} b_{10,0}-2 b_{01,1} b_{01,0}
$$

и квадратичному члену

$$
0=b_{10,1}^{2}-b_{01,1}^{2} .
$$

Последнее равенство немедленно дает $b_{10,1}= \pm b_{01,1}$. Рассмотрим, например, случай $b_{10,1}=-b_{01,1}=b$. Тогда

$$
\begin{aligned}
& 4 b_{00,0}=b_{10,0}^{2}-b_{01,0}^{2}, \\
& 2 b_{00,1}=-b\left(b_{10,0}+b_{01,0}\right),
\end{aligned}
$$

или в параметрическом виде

$$
\begin{array}{llrl}
b_{01,0}=\sqrt{2} t_{1} \operatorname{sh} t_{2}, & b_{10,0}=\sqrt{2} t_{1} \operatorname{ch} t_{2}, & b_{00,0}=\frac{1}{2} t_{1}^{2}, \\
b_{00,1}=-\sqrt{2} t_{1} t_{3}\left(\operatorname{sh} t_{2}+\operatorname{ch} t_{2}\right), & b_{10,1}=t_{3}, & b_{01,1}=-t_{3},
\end{array}
$$

где $t_{1}, t_{2}, t_{3} \in \mathbb{R}$ - произвольные вешественные параметры. Варьируя эти параметры, можно получить коэффициенты $b_{01,0}, b_{10,0}, \ldots, b_{00,0}$ и, следовательно, коэффициенты исходного оператора $a_{10}, a_{01}, a_{00}$ так, чтобы они принадлежали некоторому наперед заданному полю. Например, если $t_{1}, t_{3} \in \mathbb{Q}$ и $t_{2}=0$, то

$$
\begin{aligned}
& b_{01,0}=0, \quad b_{10,0}=\sqrt{2} t_{1}, \quad b_{00,0}=\frac{1}{2} t_{1}^{2}, \\
& b_{00,1}=-\sqrt{2} t_{1} t_{3}, \quad b_{10,1}=t_{3}, \quad b_{01,1}=-t_{3},
\end{aligned}
$$

и мы получаем двухпараметрическую факторизацию в поле $\mathbb{Q}(\sqrt{2})$ :

$$
A_{2}=\left(\partial_{x}+\partial_{y}+\frac{2 t_{3} x+\sqrt{2} t_{1}}{2}\right)\left(\partial_{x}-\partial_{y}+\frac{\sqrt{2} t_{1}}{2}\right) .
$$

Случай $b_{10,0}=b_{01,0}$ может быть рассмотрен аналогично предыдущему, а случай 2 аналогично случаю 1 . Объединяя все результаты, мы получим описание всех допускающих факторизацию ЛДО в частных производных вида (26) с соответствуюшими ограничениями на коэффициенты.

\section{6. ЗАКЛЮЧЕНИЕ}

В настояшей работе представлена процедура исключаюшей порядковой редукции для явной факторизации Л ДО в частных производных произвольного порядка. Показано, что в случае общего положения, т.е. когда характеристический полином имеет простой корень, эта процедура является полностью алгебраической и не требует решения дифференциального уравнения. В противном случае задача факторизации эквивалентна решению некоторого уравнения Риккати. Также показано, что ЛДО в частных производных различных типов обладают различными факторизационными свойствами, а именно алгебраическая факторизация гиперболических операторов, когда она возможна, может быть осушествлена над полем $\mathbb{R}$, в то время как факторизация эллиптических операторов возможна только над полем $\mathbb{C}$. В случае параболического оператора алгебраическая факторизация невозможна. 
Благодарности. Авторы благодарны организационному комитету конференции "Интегрируемые системы", которые предоставили прекрасную возможность для встреч и совместной работы. Мы также очень признательны Centro Internacional de Ciencias (Куэрнавака, Мексика), где была сделана бо́льшая часть этой работы, за гостеприимство. Мы выражаем особую благодарность А.Б. Шабату и С.П. Цареву за весьма стимулирующие дискуссии в процессе написания этой работы. Е. А. Карташева благодарит Austrian Science Foundation (FWF) за поддержку в рамках проектов SFB F013/F1304.

\section{Список литературы}

[1] J. Apel. J. Symb. Comp. 1998. V. 25. Р. 683-704; П. Олвер. Приложения групп Ли к дифференциальным уравнениям. М.: Мир, 1989; J. F. Pommaret. Partial differential equations and Lie pseudogroups. N.Y.: Gordon and Breach, 1978.

[2] M. Bronstein. An improved algorithm for factoring linear ordinary differential operators. In: Proc. of the Int. Symp. on Symbolic and Algebraic Computation (ISSAC'94). Ed. J. von zur Gathen, M. Giesbrecht. Baltimore, MD: ACM Press, 1994. P. 336-340; F. Schwarz. A factorization algorithm for linear ordinary differential equations. In: Proc. of the Int. Symp. on Symbolic and Algebraic Computation (ISSAC'89). Ed. G.H. Gonnet. N.Y.: ACM Press, 1989. P. 17-25; S.P. Tsarev. An algorithm for complete enumeration of all factorizations of a linear ordinary differential operator. In: Proc. of the Int. Symp. on Symbolic and Algebraic Computation (ISSAC'96). Ed. Y. N. Lakshman. N.Y.: ACM Press, 1996. P. 226-231.

[3] E. Beke. Math. Ann. 1894. V. 45. P. 278-300.

[4] A. Loewy. Math. Ann. 1906. V. 62. P. 89-117; 1903. V. 56. P. 549-584.

[5] E. Landau. J. für Math. 1901. V. 124. P. 115-120.

[6] D. Grigoriev, F. Schwarz. Computing. 2004. V. 73. P. 179-197.

[7] S. P. Tsarev. Factorization of linear partial differential operators and Darboux integrability of nonlinear PDEs. In: Poster at ISSAC'98, 1998; cs.SC/9811002.

[8] S. P. Tsarev. Factorization of overdetermined systems of linear partial differential equations with finite dimensional solution space. In: Proc. 4th Int. Workshop on Computer Algebra Scient. Comput. (CASC-2001). Eds. V. Ganzha, E. Mayr, V. Vorozhtsov. Berlin: Springer, 2001. P. 529-539; Z. Li, F. Schwarz, S. P. Tsarev. J. Symb. Comput. 2003. V. 36. P. 443-471.

Поступила в редакцию 28.I.2005 г. 